\title{
DMTCP: Bringing Checkpoint-Restart to Python
}

\author{
Kapil Arya ${ }^{\ddagger *}$, Gene Cooperman \\ http://www.youtube.com/watch?v=11_wGZ z 0 JEE
}

\begin{abstract}
DMTCP (Distributed MultiThreaded CheckPointing) is a mature checkpoint-restart package. It operates in user-space without kernel privilege, and adapts to application-specific requirements through plugins. While DMTCP has been able to checkpoint Python and IPython "from the outside" for many years, a Python module has recently been created to support DMTCP. IPython support is included through a new DMTCP plugin. A checkpoint can be requested interactively within a Python session, or under the control of a specific Python program. Further, the Python program can execute specific Python code prior to checkpoint, upon resuming (within the original process), and upon restarting (from a checkpoint image). Applications of DMTCP are demonstrated for: (i) Python-based graphics using VNC; (ii) a Fast/Slow technique to use multiple hosts or cores to check one Cython computation in parallel; and (iii) a reversible debugger, $\mathrm{FReD}$, with a novel reverse-expression watchpoint feature for locating the cause of a bug.
\end{abstract}

Index Terms—checkpoint-restart, DMTCP, IPython, Cython, reversible debugger

\section{Introduction}

DMTCP (Distributed MultiThreaded CheckPointing) [Ansel09] is a mature user-space checkpoint-restart package. One can view checkpoint-restart as a generalization of pickling. Instead of saving an object to a file, one saves the entire Python session to a file. Checkpointing graphics in Python is also supported-by checkpointing a virtual network client (VNC) session with Python running inside that session.

DMTCP is available as a Linux package for many popular Linux distributions. DMTCP can checkpoint Python or IPython from the outside, i.e. by treating Python as a black box. To enable checkpointing, the Python interpreter is launched in the following manner:

\$ dmtcp_checkpoint python <args>

\$ dmtcp_command--checkpoint

The command dmtcp_command can be used at any point to create a checkpoint of the entire session.

However, most Python programmers will prefer to request a checkpoint interactively within a Python session, or else programmatically from inside a Python or Cython program.

DMTCP is made accessible to Python programmers as a Python module. Hence, a checkpoint is executed as import

*Corresponding author: kapil@ccs.neu.edu

* Northeastern University

Copyright $(02013$ Kapil Arya et al. This is an open-access article distributed under the terms of the Creative Commons Attribution License, which permits unrestricted use, distribution, and reproduction in any medium, provided the original author and source are credited. dmtcp; dmtcp.checkpoint(). This Python module provides this and other functions to support the features of DMTCP. The module for DMTCP functions equally well in IPython.

This DMTCP module implements a generalization of a saveWorkspace function, which additionally supports graphics and the distributed processes of IPython. In addition, three novel uses of DMTCP for helping debug Python are discussed.

1) Fast/Slow Computation-Cython provides both traditional interpreted functions and compiled $\mathrm{C}$ functions. Interpreted functions are slow, but correct. Compiled functions are fast, but users sometimes declare incorrect $\mathrm{C}$ types, causing the compiled function silently return a wrong answer. The idea of fast/slow computation is to run the compiled version on one computer node, while creating checkpoint images at regular intervals. Separate computer nodes are used to check each interval in interpreted mode between checkpoints.

2) FReD—a Fast Reversible Debugger that works closely with the Python pdb debugger, as well as other Python debuggers.

3) Reverse Expression Watchpoint-This is a novel feature within the FReD reversible debugger. Assume a bug occurred in the past. It is associated with the point in time when a certain expression changed. Bring the user back to a pdb session at the step before the bug occurred.

The remaining sections describe: the DMTCP-Python Integration through a Python Module; and several extensions of the integration of DMTCP with Python. The extensions include support for Checkpointing Python-Based Graphics; Checking Cython with Multiple CPython Instances (fast/slow technique); and Reversible Debugging with FReD. More information about DMTCP is added in Appendix: Background of DMTCP.

\section{DMTCP-Python Integration through a Python Module}

A Python module, dmt cp. py, has been created to support checkpointing both from within an interactive Python/IPython session and programmatically from within a Python or Cython program. DMTCP has been able to asynchronously generate checkpoints of a Python session for many years. However, most users prefer the more fine-grained control of a Python programmatic interface to DMTCP. This allows one to avoid checkpointing in the middle of a communication with an external server or other atomic transaction.

\section{A Python Module to Support DMTCP}

Some of the features of module.py are best illustrated through an example. Here, a checkpoint request is made from within the application. 


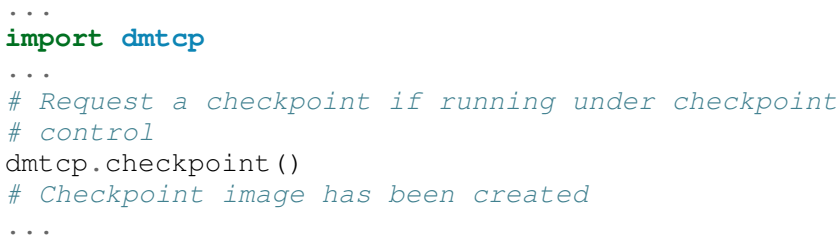

It is also easy to add pre- and post-checkpoint processing actions.

$\cdots$

import dmtcp

..

def my_ckpt (<args $>)$ :

\# Pre processing

my_pre_ckpt_hook (<args $>)$

$\cdots$

\# Create checkpoint

dmtcp. checkpoint ()

* checkpoint image has been created

.

if dmtcp.isResume ():

\# The process is resuming from a checkpoint

my_resume_hook (<args $>$ )

else:

\# The process is restarting from a previous

\# checkpoint

my_restart_hook (<args $>$ )

...

return

The function my_ckpt can be defined in the application by the user and can be called from within the user application at any point.

\section{Extending the DMTCP Module for Managing Sessions}

These core checkpoint-restart services are further extended to provide the user with the concept of multiple sessions. A checkpointed Python session is given a unique session id to distinguish it from other sessions. When running interactively, the user can view the list of available checkpointed sessions. The current session can be replaced by any of the existing session using the session identifier.

The application can programmatically revert to an earlier session as shown in the following example:

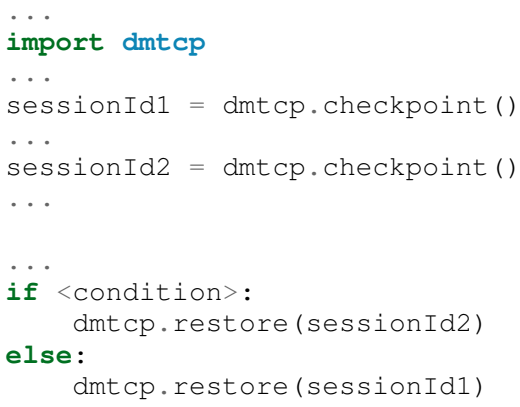

\section{Save-Restore for IPython Sessions}

To checkpoint an IPython session, one must consider the configuration files. The configuration files are typically stored in user's home directory. During restart, if the configuration files are missing, the restarted computation may fail to continue. Thus, DMTCP must checkpoint and restore all the files required for proper restoration of an IPython session.
Attempting to restore all configuration files during restart poses yet another problem: the existing configuration files might have newer contents. Overwriting these newer files with copies from the checkpoint time may result in the loss of important changes.

To avoid overwriting the existing configuration files, the files related to IPython session are restored in a temporary directory. Whenever IPython shell attempts to open a file in the original configuration directory, the filepath is updated to point to the temporary directory. Thus, the files in the original configuration directory are never modified. Further, the translation from original to temporary path is transparent to the IPython shell.

\section{Save-Restore for Parallel IPython Sessions}

DMTCP is capable of checkpointing a distributed computations with processes running on multiple nodes. It automatically checkpoints and restores various kinds of inter-process communication mechanisms such as shared-memory, message queues, pseudottys, pipes and network sockets.

An IPython session involving a distributed computation running on a cluster is checkpointed as a single unit. DMTCP allows restarting the distributed processes in a different configuration than the original. For example, all the processes can be restarted on a single computer for debugging purposes. In another example, the computation may be restarted on a different cluster altogether.

\section{Checkpointing Python-Based Graphics}

Python is popular for scientific visualizations. It is possible to checkpoint a Python session with active graphics windows by using VNC. DMTCP supports checkpoint-restart of VNC server. In this case, a VNC server can be started automatically. The process environment is modified to allow the Python interpreter to communicate with the VNC server instead of the $\mathrm{X}$-window server. For visualization, a VNC client can be fired automatically to display the graphical window. During checkpoint, the VNC server is checkpointed as part of the computation, while the VNC client is not. During restart, the Python session and the VNC server are restored from their checkpoint images, and a fresh VNC client is launched. This VNC client communicates with the restored server and displays the graphics to the end user.

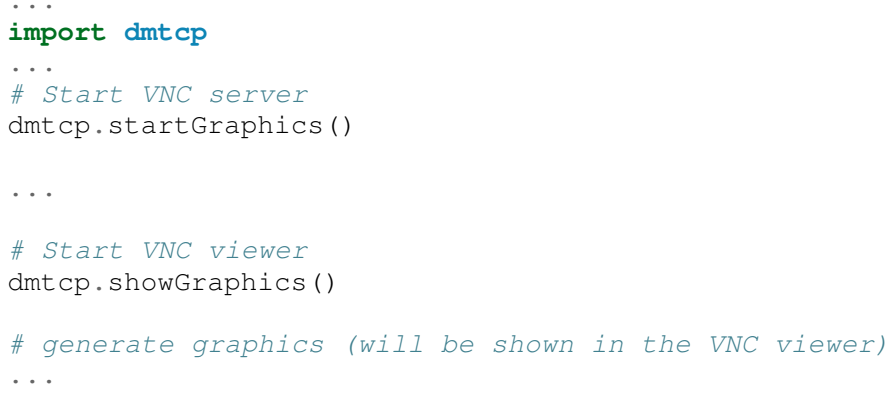

To understand the algorithm behind the code, we recall some VNC concepts. X-window supports multiple virtual screens. A VNC server creates a new virtual screen. The graphics contained in the VNC server is independent of any $\mathrm{X}$-window screen. The VNC server process persists as a daemon. A VNC viewer displays a specified virtual screen in a window in a console. When python generates graphics, the graphics is sent to a virtual screen specified by the environment variable \$DISPLAY.

The command dmtcp.startGraphics () creates a new $\mathrm{X}$-window screen by creating a new VNC server and sets the 


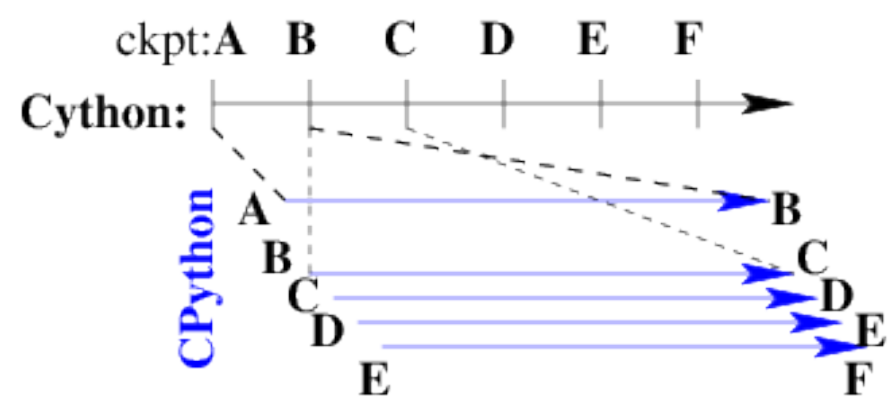

Fig. 1: Fast Cython with Slow CPython "checking" nodes.

\$DISPLAY environment variable to the new virtual screen. All python graphics are now sent to this new virtual screen. The additional screen is invisible to the python user until the python command dmtcp.showgraphics() is given. The Python Command dmtcp. showGraphics () operates by invoking a VNC viewer.

At the time of checkpoint, the VNC server process is checkpointed along with the python interpretor while the $\mathrm{VNC}$ viewer is not checkpointed.

On restart, the VNC server detects the stale connection to the old VNC viewers. The VNC server perceives this as the VNC viewer process that has now died. The DMTCP module then launches anew VNC viewer to connect to the VNC server.

\section{Checking Cython with Multiple CPython Instances}

A common problem for compiled versions of Python such as Cython [Behnel10] is how to check whether the compiled computation is faithful to the interpreted computation. Compilation errors can occur if the compiled code assumes a particular $\mathrm{C}$ type, and the computation violates that assumption for a particular input. Thus, one has to choose between speed of computation and a guarantee that that the compiled computation is faithful to the interpreted computation.

A typical scenario might be a case in which the compiled Cython version ran for hours and produced an unexpected answer. One wishes to also check the answer in a matter of hours, but pure Python (CPython) would take much longer.

Informally, the solution is known as a fast/slow technique. There is one fast process (Cython), whose correctness is checked by multiple slow processes (CPython). The core idea is to run the compiled code, while creating checkpoint images at regular intervals. A compiled computation interval is checked by copying the two corresponding checkpoints (at the beginning and end of the interval) to a separate computer node for checking. The computation is restarted from the first checkpoint image, on the checking node. But when the computation is first restarted, the variables for all user Python functions are set to the interpreted function object. The interval of computation is then re-executed in interpreted mode until the end of the computation interval. The results at the end of that interval can then be compared to the results at the end of the same interval in compiled mode.

Figure 1 illustrates the above idea. A similar idea has been used by [Ghoshal11] for distributed speculative parallelization.

Note that in order to compare the results at the end of a computation interval, it is important that the interpreted version on the checker node stop exactly at the end of the interval, in order to compare with the results from the checkpoint at the end of the same interval. The simplest way to do this is to add a counter to a frequently called function of the end-user code. The counter is incremented each time the function is called. When the counter reaches a pre-arranged multiple (for example, after every million calls), the compiled version can generate a checkpoint and write to a file the values of variables indicating the state of the computation. The interpreted version writes to a file the values of variables indicating its own state of the computation.

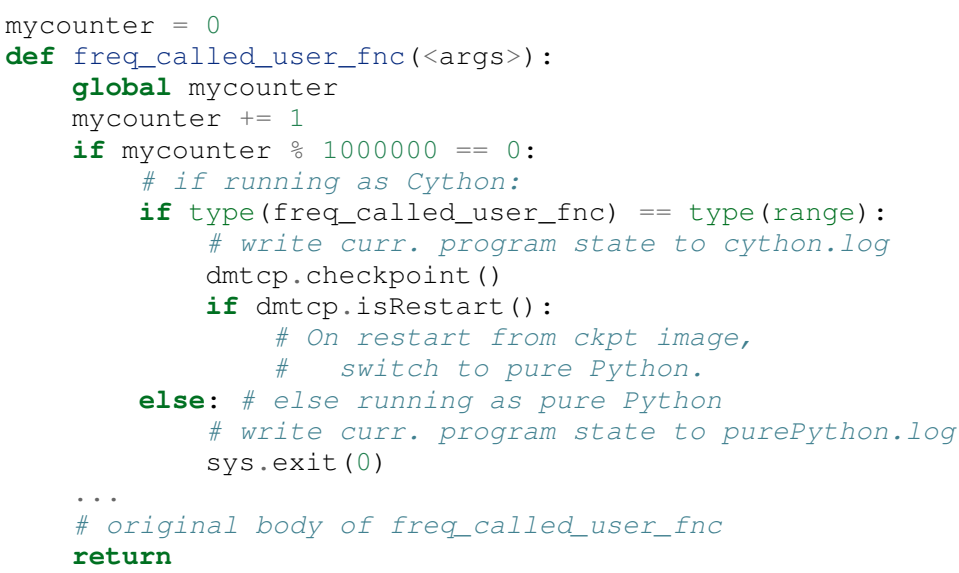

The above code block illustrates the principles. One compares cython.log and purePython.log to determine if the compiled code was faithful to the interpreted code. If the Cython code consists of direct $\mathrm{C}$ calls between functions, then it will also be necessary to modify the functions of the $\mathrm{C}$ code generated by Cython, to force them to call the pure Python functions on restart after a checkpoint.

\section{Reversible Debugging with FReD}

While debugging a program, often the programmer over steps and has to restart the debugging session. For example, while debugging a program, if the programmer steps over (by issue next command inside the debugger) a function $f()$ only to determine that the bug is in function $f()$ itself, he or she is left with no choice but to restart from the beginning.

Reversible debugging is the capability to run an application "backwards" in time inside a debugger. If the programmer detects that the problem is in function $f()$, instead of restarting from the beginning, the programmer can issue a reverse-next command which takes it to the previous step. He or she can then issue a step command to step into the function in order to find the problem.

FReD (Fast Reversible Debugger) [Arya12], [FReD13] is a reversible debugger based on checkpoint-restart. FReD is implemented as a set of Python scripts and uses DMTCP to create checkpoints during the debugging session. FReD also keeps track of the debugging history. Figure 2 shows the architecture of FReD.

\section{A Simple UNDO Command}

The $U N D O$ command reverses the effect of a previous debugger command such as next, continue or finish. This is the most basic of reversible debugging commands.

The functionality of the UNDO command for debugging Python is trivially implemented. A checkpoint is taken at the beginning of the debugging session and a list of all debugging commands issued since the checkpoint are recorded. 


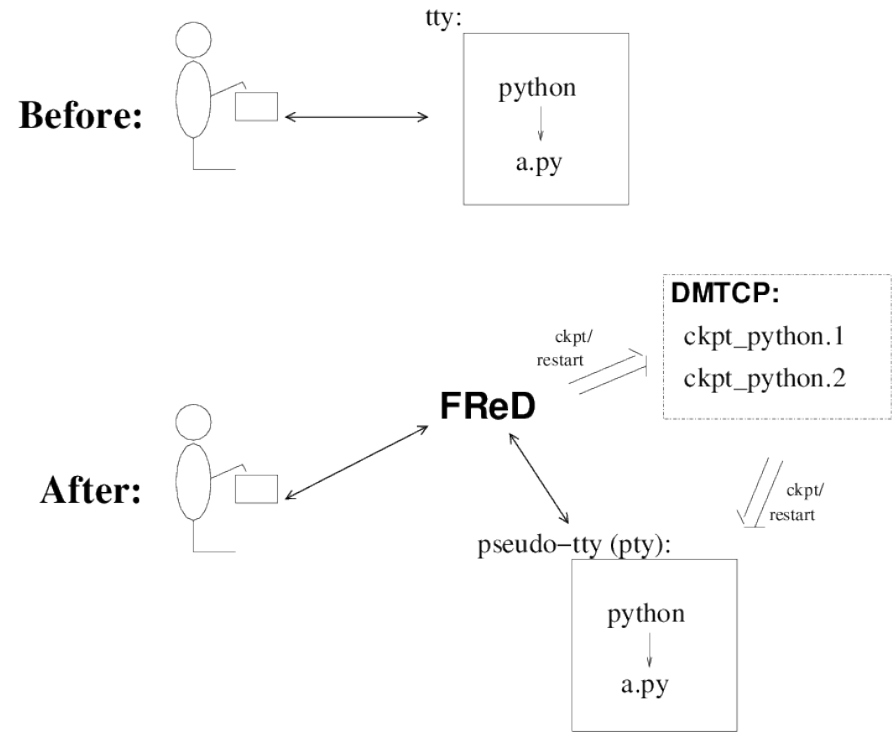

Fig. 2: Fast Reversible Debugger.

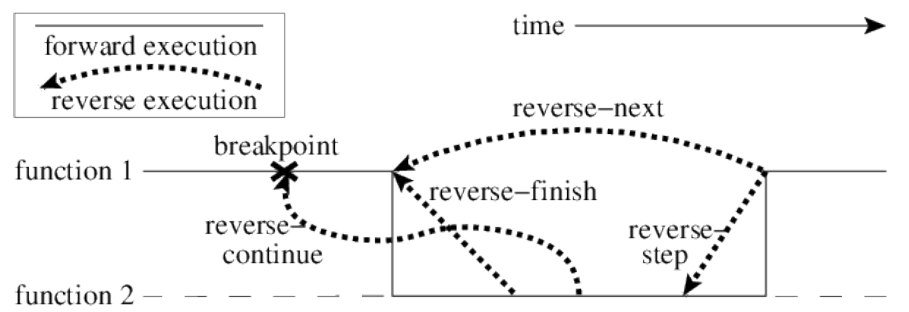

Fig. 3: Reverse Commands.

To execute the UNDO command, the debugging session is restarted from the checkpoint image, and the debugging commands are automatically re-executed from the list excluding the last command. This takes the process back to before the debugger command was issued.

In longer debugging sessions, checkpoints are taken at frequent intervals to reduce the time spent in replaying the debugging history.

\section{More complex reverse commands}

Figure 3 shows some typical debugging commands being executed in forward as well as backward direction in time.

Suppose that the debugging history appears as [next, next] i.e. the user issued two next commands. Further, the second next command stepped over a function $\mathrm{f}($ ). Suppose further that FReD takes checkpoints before each of these commands. In this situation, the implementation for reverse-next command is trivial: one restarts from the last checkpoint image. However, if the command issued were reverse-step, simply restarting from the previous checkpoint would not suffice.

In this last case, the desired behavior is to take the debugger to the last statement of the function $f()$. In such a situation one needs to decompose the last command into a series of commands. At the end of this decomposition, the last command in the history is a step. At this point, the history may appear as: [next, step, next, ..., next, step]. The process is then restarted from the last checkpoint and the debugging history is executed excluding the last step command. Decomposing a

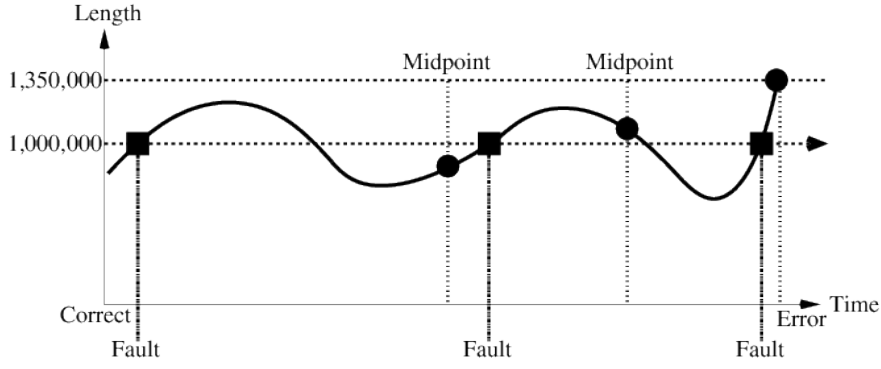

Fig. 4: Reverse Expression Watchpoint.

command into a series of commands terminating with step is non-trivial, and an algorithm for that decomposition is presented in [Visan11] .

A typical debugging session in FReD with Python:

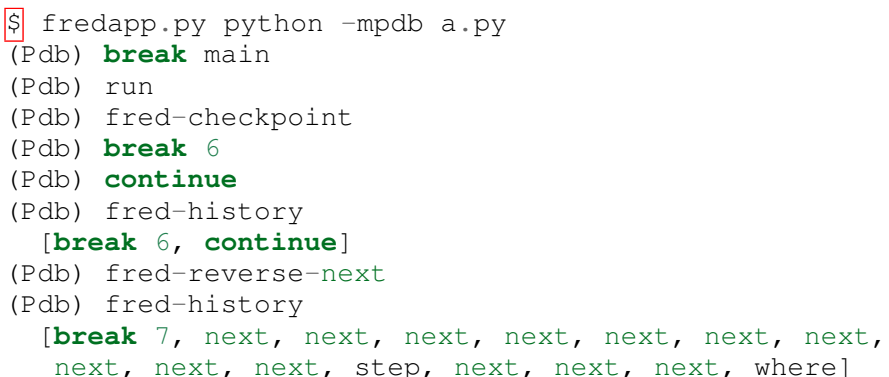

\section{Reverse Expression Watchpoints}

The reverse expression watchpoint automatically finds the location of the fault for a given expression in the history of the program execution. It brings the user directly to a statement (one that is not a function call) at which the expression is correct, but executing the statement will cause the expression to become incorrect.

Figure 4 provides a simple example. Assume that a bug occurs whenever a linked list has length longer than one million. So an expression linked_list.len() $<=1000000$ is assumed to be true throughout. Assume that it is too expensive to frequently compute the length of the linked list, since this would require $O\left(n^{2}\right)$ time in what would otherwise be a $O(n)$ time algorithm. (A more sophisticated example might consider a bug in an otherwise duplicate-free linked list or an otherwise cycle-free graph. But the current example is chosen for ease of illustrating the ideas.)

If the length of the linked list is less than or equal to one million, we will call the expression "good". If the length of the linked list is greater than one million, we will call the expression "bad". A "bug" is defined as a transition from "good" to "bad". There may be more than one such transition or bug over the process lifetime. Our goal is simply to find any one occurrence of the bug.

The core of a reverse expression watchpoint is a binary search. In Figure 4, assume a checkpoint was taken near the beginning of the time interval. So, we can revert to any point in the illustrated time interval by restarting from the checkpoint image and reexecuting the history of debugging commands until the desired point in time.

Since the expression is "good" at the beginning of Figure 4 and it is "bad" at the end of that figure, there must exist a buggy statement-a statement exhibiting the transition from "good" to "bad". A standard binary search algorithm converges to a case in which the current statement is "good" and the next statement 
transitions from "good" to "bad". By the earlier definition of a "bug", FReD has found a statement with a bug. This represents success.

If implemented naively, this binary search requires that some statements may need to be re-executed up to $\log _{2} N$ times. However, FReD can also create intermediate checkpoints. In the worst case, one can form a checkpoint at each phase of the binary search. In that case, no particular sub-interval over the time period needs to be executed more than twice.

A typical use of reverse-expression-watchpoint:

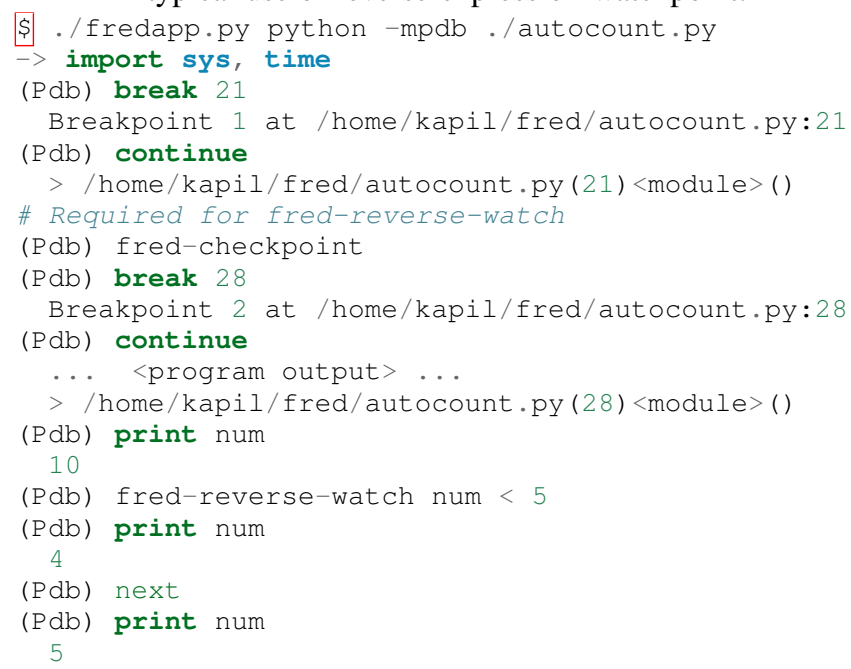

\section{Conclusion}

DMTCP is a widely used standalone checkpoint-restart package. We have shown that it can be closely integrated with Python. Specifically, parallel sessions with IPython, alternating interpreted and compiled execution modes, graphics, and enhancing Python debugger with reversibility. The implementation can be extended by the end users to augment the capabilities of Python beyond the simple example of checkpoint-restart.

\section{Acknowledgment}

This work was partially supported by the National Science Foundation under Grant OCI-0960978.

\section{Appendix: Background of DMTCP}

DMTCP [Ansel09] is a transparent checkpoint-restart package with its roots going back eight years [Rieker06]. It works completely in user space and does not require any changes to the application or the operating system. DMTCP can be used to checkpoint a variety of user applications including Python.

Using DMTCP to checkpoint an application is as simple as executing the following commands:

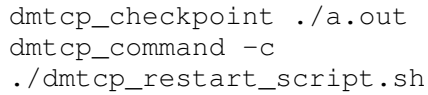

DMTCP automatically tracks all local and remote child processes and their relationships.

As seen in Figure 5, a computation running under DMTCP consists of a centralized coordinator process and several user processes. The user processes may be local or distributed. User processes may communicate with each other using sockets, sharedmemory, pseudo-terminals, etc. Further, each user process has a checkpoint thread which communicates with the coordinator.

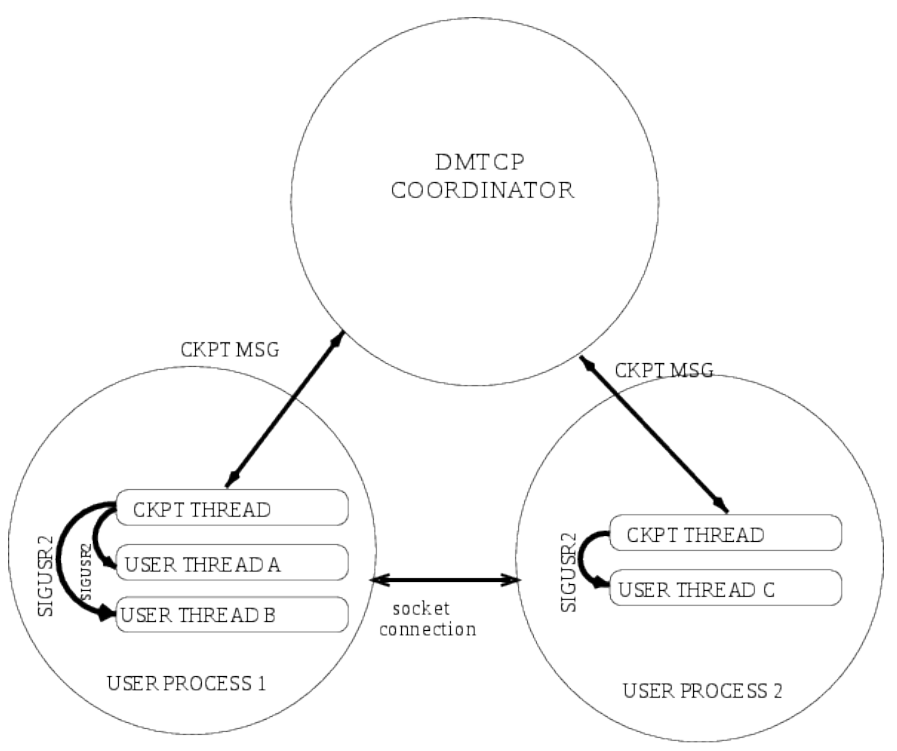

Fig. 5: Architecture of DMTCP.

\section{DMTCP Plugins}

DMTCP plugins are used to keep DMTCP modular. There is a separate plugin for each operating system resource. Examples of plugins are pid plugin, socket plugin, and file plugin. Plugins are responsible for checkpointing and restoring the state of their corresponding resources.

The execution environment can change between checkpoint and restart. For example, the computation might be restarted on a different computer which has different file mount points, a different network address, etc. Plugins handle such changes in the execution environment by virtualizing these aspects. Figure 6 shows the layout of DMTCP plugins within the application.

\section{DMTCP Coordinator}

DMTCP uses a stateless centralized process, the DMTCP coordinator, to synchronize checkpoint and restart between distributed processes. The user interacts with the coordinator through the console to initiate checkpoint, check the status of the computation, kill the computation, etc. It is also possible to run the coordinator as a daemon process, in which case, the user may communicate with the coordinator using the command dmtcp_command.

\section{Checkpoint Thread}

The checkpoint thread waits for a checkpoint request from the DMTCP coordinator. On receiving the checkpoint request, the checkpoint thread quiesces the user threads and creates the checkpoint image. To quiesce user threads, it installs a signal handler for a dedicated POSIX signal (by default, SIGUSR2). Once the checkpoint image has been created, the user threads are allowed to resume executing application code. Similarly, during restart, once the process memory has been restored, the user threads can resume executing application code.

\section{Checkpoint}

On receiving the checkpoint request from the coordinator, the checkpoint thread sends the checkpoint signal to all the user threads of the process. This quiesces the user threads by forcing them to block inside a signal handler, defined by the DMTCP. The checkpoint image is created by writing all of user-space memory 


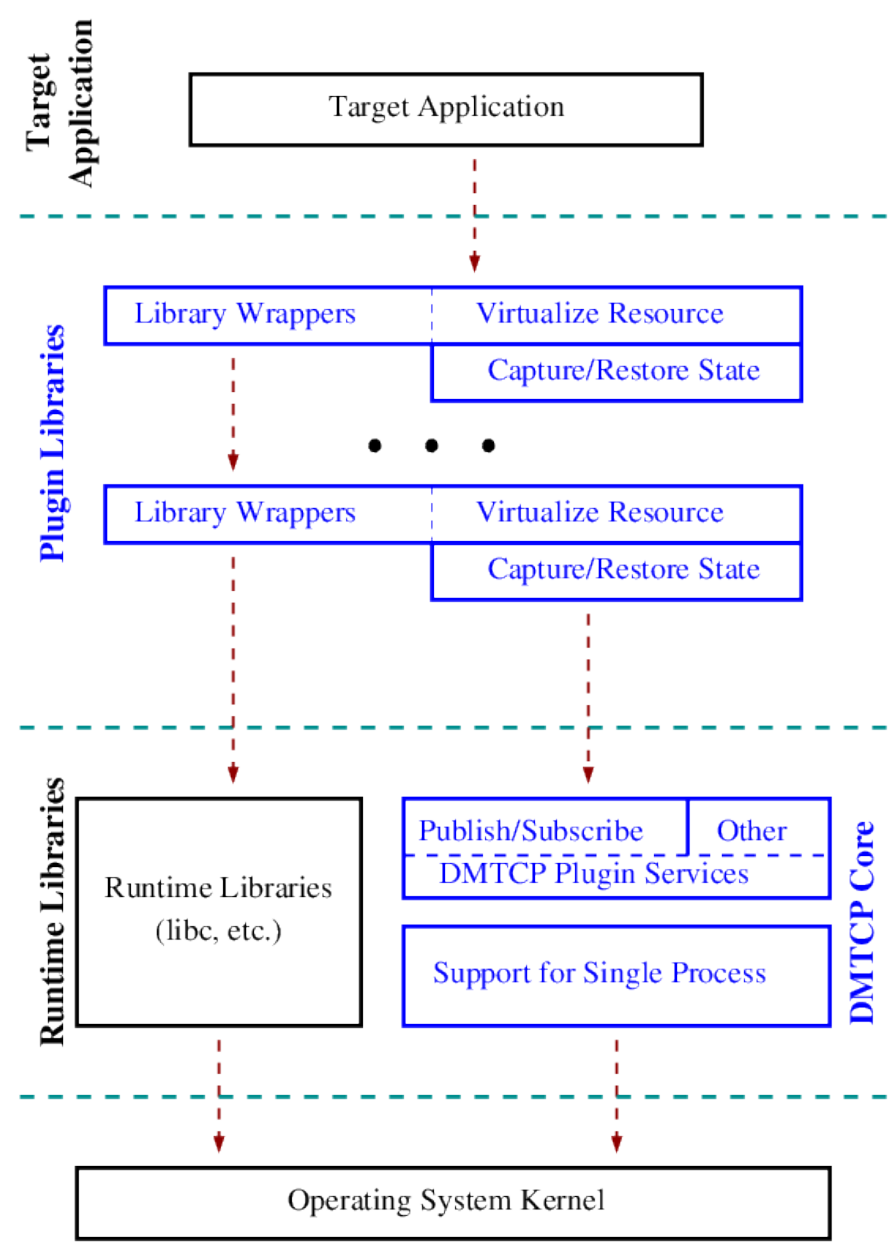

Fig. 6: DMTCP Plugins.

to a checkpoint image file. Each process has its own checkpoint image. Prior to checkpoint, each plugin will have copied into user-space memory any kernel state associated with its concerns. Examples of such concerns include network sockets, files, and pseudo-terminals. Once the checkpoint image has been created, the checkpoint thread "un-quiesces" the user threads and they resume executing application code.

At the time of checkpoint, all of user-space memory is written to a checkpoint image file. The user threads are then allowed to resume execution. Note that user-space memory includes all of the run-time libraries (libc, libpthread, etc.), which are also saved in the checkpoint image.

In some cases, state outside the kernel must be saved. For example, in handling network sockets, data in flight must be saved. This is done by draining the network data by sending a special cookie through the "send" end of each socket in one phase. In a second phase, after a global barrier, data is read from the "receive" end of each socket until the special cookie is received. The in-flight data has now been copied into user-space memory, and so will be included in the checkpoint image. On restart, the network buffers are refilled by sending the in-flight data back to the peer process, which then sends the data back into the network.

\section{Restart}

As the first step of restart phase, all memory areas of the process are restored. Next, the user threads are recreated. The plugins then receive the restart notification and restore their underlying resources, translation tables, etc. Finally, the checkpoint thread "un-quiesces" the user threads and the user threads resume executing application code.

\section{REFERENCES}

[Ansel09] Jason Ansel, Kapil Arya, and Gene Cooperman. DMTCP: Transparent Checkpointing for Cluster Computations and the Desktop, 23rd IEEE International Symposium on Parallel and Distributed Processing (IPDPS-09), 1-12, $2009 \mathrm{http}: / / \mathrm{dmtcp}$ sourceforge. net/.

[Arya12] Kapil Arya, Tyler Denniston, Ana Maria Visan, and Gene Cooperman. FReD: Automated Debugging via Binary Search through a Process Lifetime, http://arxiv.org/abs/1212.5204.

[FReD13] FReD (Fast Reversible Debugger) Software. https://github.com/ fred-dbg/fred

[Behnel10] R. Bradshaw, S. Behnel, D. S. Seljebotn, G. Ewing, et al. Cython: The Best of Both Worlds, Computing in Science Engineering, 2010.

[Ghoshal11] Devarshi Ghoshal, Sreesudhan R. Ramkumar, and Arun Chauhan. Distributed Speculative Parallelization using Checkpoint Restart, Procedia Computer Science, 2011.

[Rieker06] Michael Rieker, Jason Ansel, and Gene Cooperman. Transparent User-Level Checkpointing for the Native POSIX Thread Library for Linux, Proceeding of PDPTA-06, 492-498, 2006.

[Visan11] Ana-Maria Visan, Kapil Arya, Gene Cooperman, and Tyler Denniston. URDB: A Universal Reversible Debugger Based on Decomposing Debugging Histories, In Proc. of 6th Workshop on Programming Languages and Operating Systems (PLOS'2011) (part of Proc. of 23rd ACM SOSP), 2011. 